\title{
Erratum to: TH1/TH2 Functional Imbalance After Acute Myocardial Infarction: Coronary Arterial Inflammation or Myocardial Inflammation
}

\author{
Xiang Cheng • Yu-hua Liao • Hongxia Ge • Bin Li • Jinying Zhang • \\ Jing Yuan • Min Wang • Ying Liu • Zhangqiang Guo • Jing Chen • \\ Jin Zhang • Lanjing Zhang
}

Published online: 3 June 2014

(C) Springer Science+Business Media New York 2014

\section{Erratum to: J Clin Immunol \\ DOI 10.1007/s10875-005-4088-0}

The original version of the article unfortunately contained mistakes in Fig. 1. The correct version is presented below.

The online version of the original article can be found at http://dx.doi.org/ $10.1007 / \mathrm{s} 10875-005-4088-0$.

X. Cheng $\cdot$ Y.-h. Liao $(\bowtie) \cdot$ H. Ge $\cdot$ B. Li $\cdot$ J. Zhang $\cdot$ J. Yuan $\cdot$

M. Wang $\cdot$ Y. Liu $\cdot$ Z. Guo $\cdot$ J. Chen $\cdot$ J. Zhang

Laboratory of Cardiovascular Immunology, Institute of Cardiology,

Union Hospital, Tongji Medical College of Huazhong University of

Science and Technology, Wuhan 430022, China

e-mail: liaoyh27@hotmail.com

\section{Zhang}

Department of Internal Medicine, University of Michigan Medical

School, Ann Arbor, MI 48109, USA 
A
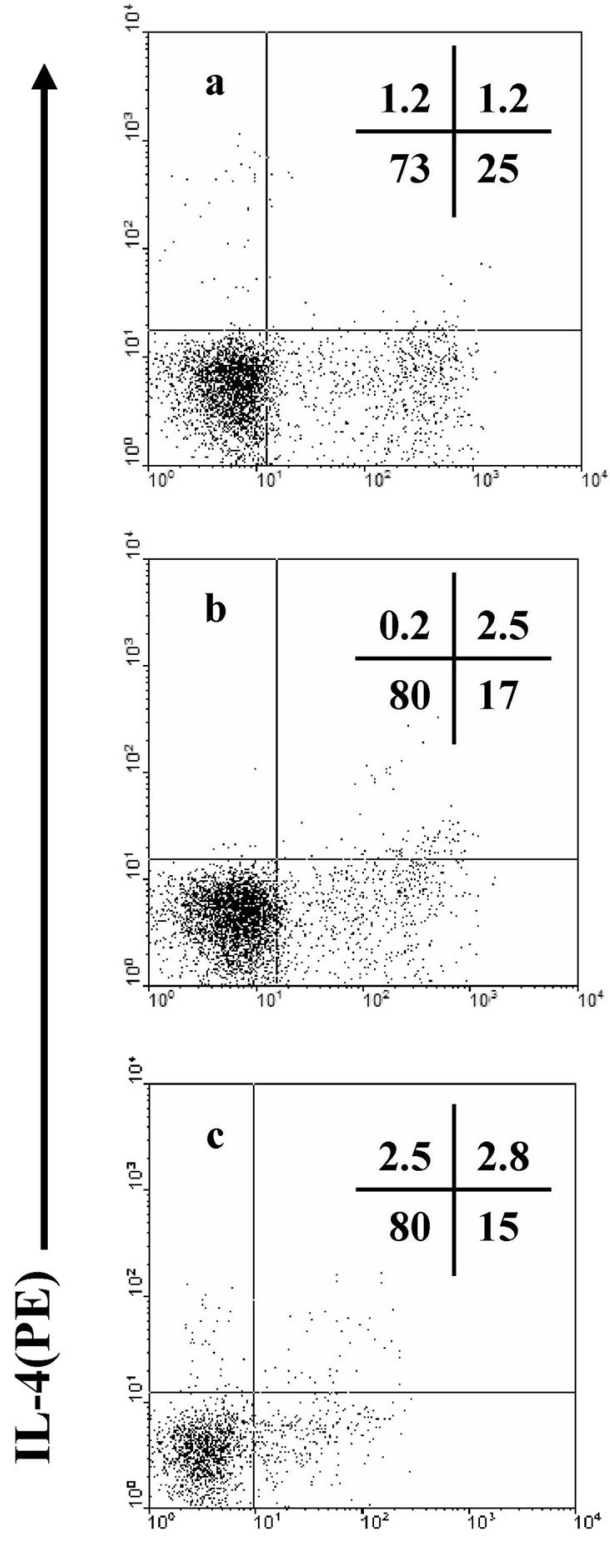

B
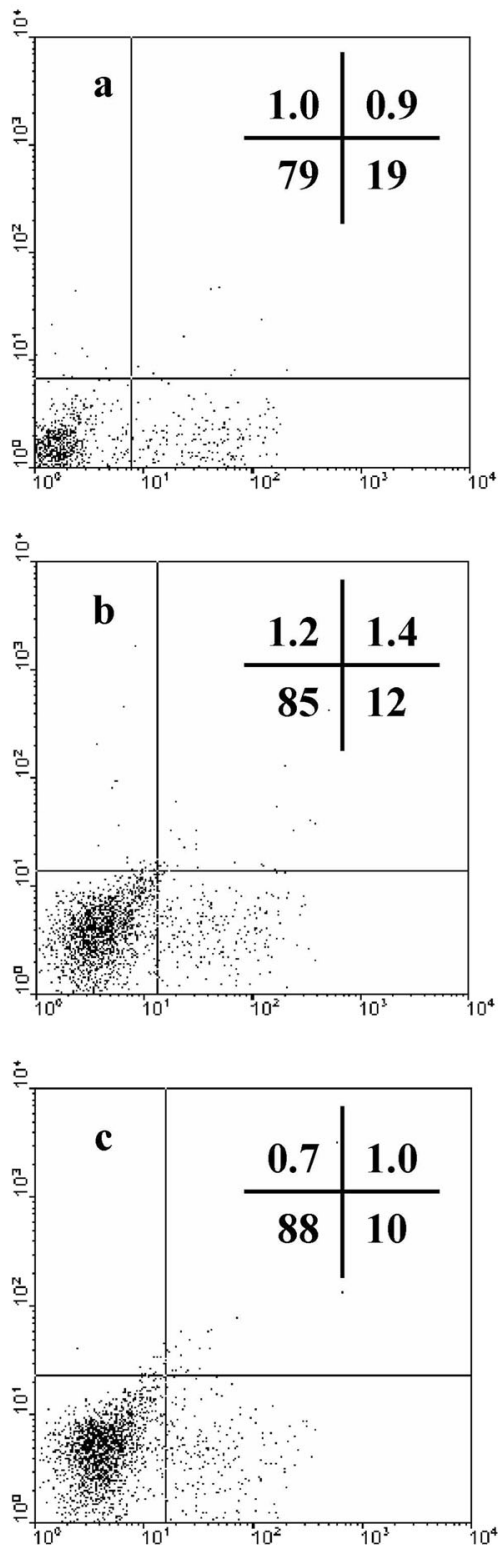

C
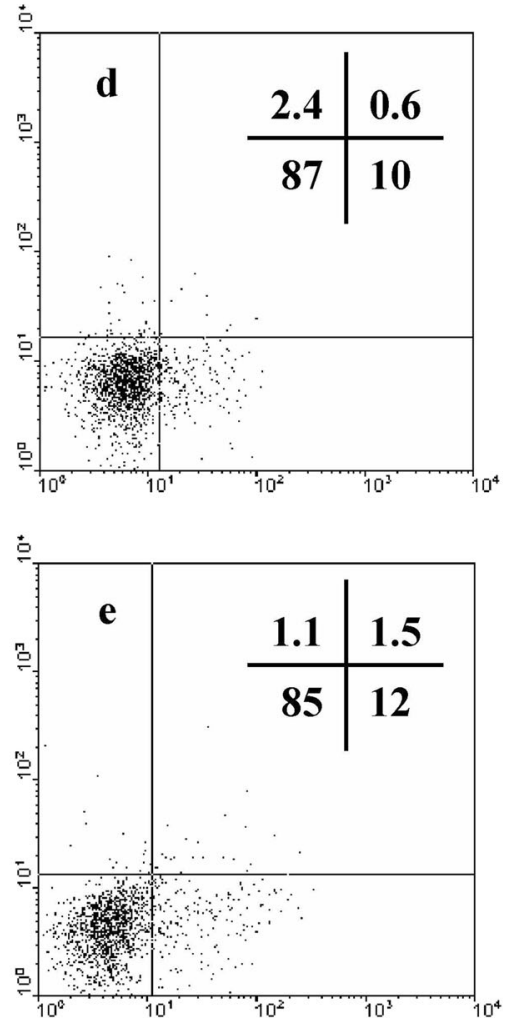

\section{IFN- $\gamma($ FITC)}

Fig. 1 The representative photographs of 3-color flow cytometric analysis of intracellular cytokines in $\mathrm{CD}^{+} \mathrm{T}$-cells (gated to CD4). Cytokineproducing T-cells in AMI (A), UA (B) and control (C) group at the different time points $((a)$, within $24 \mathrm{~h}$; (b), on day 7 and (c), on day 30 after the onset of symptom; $(d)$, group SA and (e), group CPS while coronary angiography) 